\title{
Rotor-Flying Manipulator: Modeling, Analysis, and Control
}

\author{
Bin Yang, ${ }^{1,2}$ Yuqing He, ${ }^{1}$ Jianda Han, ${ }^{1}$ and Guangjun Liu ${ }^{1,3}$ \\ ${ }^{1}$ State Key Laboratory of Robotics, Shenyang Institute of Automation, Chinese Academy of Sciences, Shenyang 110016, China \\ ${ }^{2}$ University of Chinese Academy of Sciences, Beijing 100049, China \\ ${ }^{3}$ Department of Aerospace Engineering, Ryerson University, Toronto, ON, Canada M5B 2K3
}

Correspondence should be addressed to Yuqing He; heyuqing@sia.cn

Received 14 December 2013; Revised 24 February 2014; Accepted 26 February 2014; Published 4 May 2014

Academic Editor: Rongni Yang

Copyright (C) 2014 Bin Yang et al. This is an open access article distributed under the Creative Commons Attribution License, which permits unrestricted use, distribution, and reproduction in any medium, provided the original work is properly cited.

\begin{abstract}
Equipping multijoint manipulators on a mobile robot is a typical redesign scheme to make the latter be able to actively influence the surroundings and has been extensively used for many ground robots, underwater robots, and space robotic systems. However, the rotor-flying robot (RFR) is difficult to be made such redesign. This is mainly because the motion of the manipulator will bring heavy coupling between itself and the RFR system, which makes the system model highly complicated and the controller design difficult. Thus, in this paper, the modeling, analysis, and control of the combined system, called rotor-flying multijoint manipulator (RF-MJM), are conducted. Firstly, the detailed dynamics model is constructed and analyzed. Subsequently, a full-state feedback linear quadratic regulator (LQR) controller is designed through obtaining linearized model near steady state. Finally, simulations are conducted and the results are analyzed to show the basic control performance.
\end{abstract}

\section{Introduction}

Rotor-flying robot (RFR) has been researched for several decades and achieved great development. To date, RFRs have shown their priority in many applications, such as search, rescue, and surveillance [1-6]. However, these applications are often passive. That means the RFR system can't manipulate the interested objects by a direct physical interaction.

Most recently, this problem has attained more and more attentions from many researches. For example, some researchers suggest to equip a gripper on the RFR system so that the RFR system can grasp as shown Figure 1 [7-9]. But it still has some disadvantages including that (1) the manipulation can only be implemented through controlling the attitude of the RFR system. However, the precise control of the RFR system is difficult due to its complicated dynamics, and the precise manipulation is impossible. (2) With this structure, in order to manipulate an object, the RFR must approach it. This, however, may both bring the so-called ground effect and "blow" the object, which makes the precise control much more difficult.

In this paper, a new system structure as shown in Figure 2 is proposed. The system is composed of an RFR system and a multijoint manipulator and thus called rotor-flying multijoint manipulator (RF-MJM). Compared to the structure in Figure 1, the multiple-joint manipulator can be used to regulate position and attitude of the end-gripper. This is very useful to compensate the control imprecision of the RFR system and makes precise manipulation much easier.

However, it is obvious that the system shown in Figure 2 is very difficult to be controlled. This is mainly because the RFR system itself is very sensitive to the external disturbance (force/moment), especially for the time varying disturbance, for example, from a moving manipulator as the new proposed RF-MJM system. Thus, it is very important and necessary for us to study the detailed model that can describe the coupling between the RFR and the manipulator. What is more, to construct a full-state high fidelity dynamics model is also a benefit for optimizing the design parameters, for example, the mass, the joint number, and the configuration of the manipulator, and testing the designed control algorithms.

Thus in this paper, the dynamics model of the RFMJM system is constructed and analyzed to show its basic performance. Moreover, the linear LQR controller is designed to test the basic closed loop performance of it. The main contributions of this paper are as the following three aspects: 


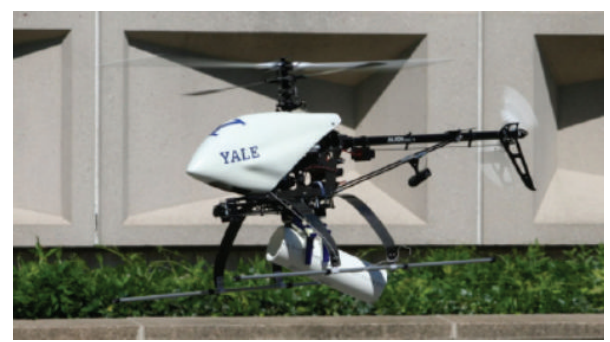

(a)

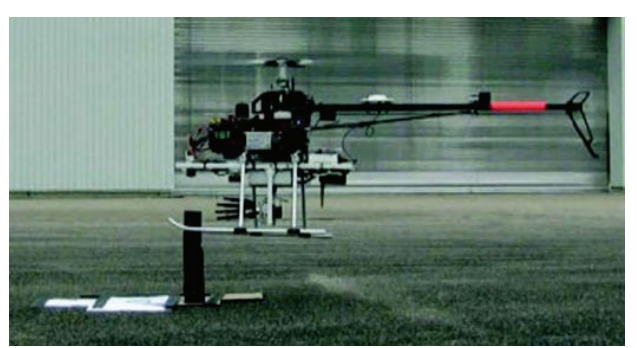

(b)

FIGURE 1: RFR system designed by Yale University (a) and DLR (b).

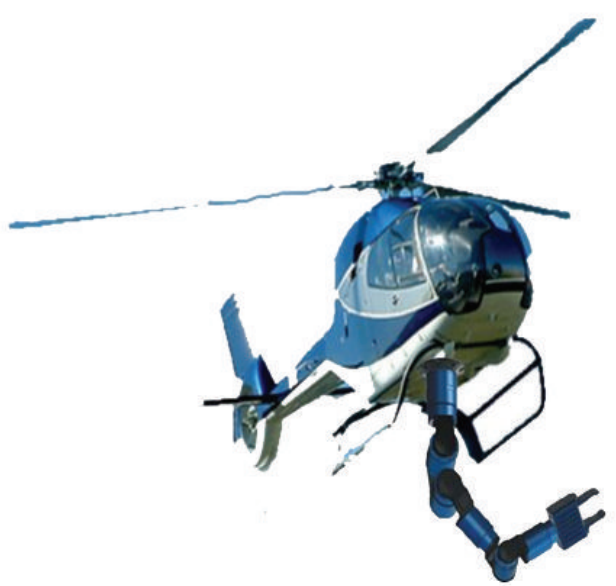

FIGURE 2: Sketch of the RF-MJM system.

(1) a detailed full-state high fidelity nonlinear dynamics model of the RF-MJM system is constructed through using Euler-Lagrange method; (2) the dynamics couplings between the RFR and the manipulator are analyzed in detail, which is good for the optimal design of the system configuration; (3) LQR controller is designed based on the linearized system model and simulations are conducted to show the basic control performance of the new proposed system.

\section{Dynamics Model of RF-MJM}

The dynamics model of the RF-MJM is composed of three parts as shown in Figure 3: the body dynamics model, the mid-dynamics model, and the actuator model. The body dynamics model describes the relation between the motion state and the external force/torque exerting on the body of the robot; the mid-dynamics model represents how the force/torque is produced, that is, the aerodynamics of the RFR, and the torque from the manipulator joint motor, while the actuator model depicts the dynamical characteristic of the actuator, for example, the motors of the manipulator and the steering engine of the RFR. For the RF-MJM system,

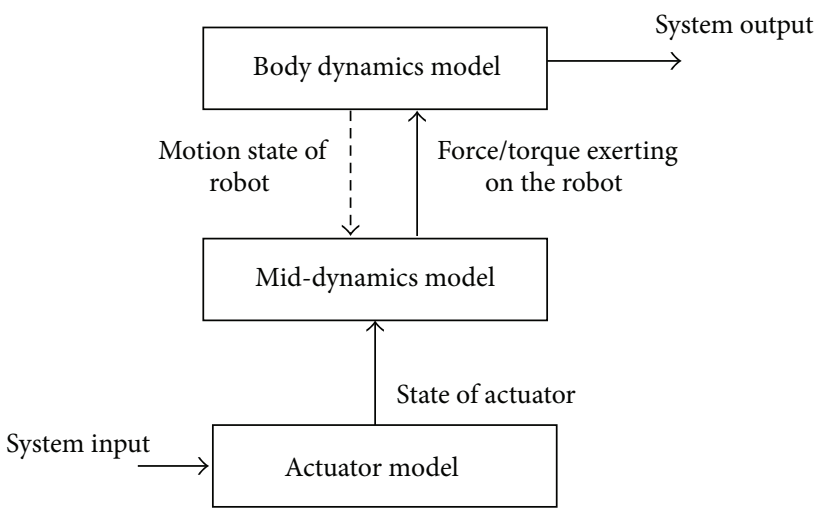

FIgURE 3: Model structure of the RF-MJM system.

the coupling between the RFR and the manipulator will mainly influence the body dynamics model, which, then, will be discussed in this paper.

A RF-MJM system is actually a multilink system shown as in Figure 4, where the cube denotes the RFR and the ellipsoids denote the link of the manipulator; $\Sigma_{0}, \Sigma_{I}, \Sigma_{E}$, and $\Sigma_{i}$ are RFR body-fixed reference frame, earth-fixed inertial frame, endgripper frame, and the frame of the $i$ th joint of manipulator; $J_{i}(i=1,2, \ldots, n)$ denote the joint of the manipulator; $p_{i}$ denotes its position vector in the frame of $\Sigma_{I} ; C_{0}$ and $C_{i}$ are the position vector of the center of mass (COM) of the link RFR and the link $i ; d_{0}$ and $d_{i}$ are the position vectors of $C_{0}$ and $C_{i} ; a_{i}$ is the vector from $J_{i}$ to $C_{i} ; b_{0}$ is the vector from $\mathrm{COM}$ of the RFR to the first joint; $b_{i}$ is vector from $C_{i}$ to $J_{i+1}$; $n$ is the number of the manipulator's link.

2.1. Kinematics Model. In this paper, we suppose that both the RFR system and the manipulator are rigid. Thus, the following geometric relations are satisfied:

$$
d_{i}=d_{0}+b_{0}+\sum_{k=1}^{i-1}\left(a_{k}+b_{k}\right)+a_{i}
$$

Differentiate it with respect to time and we have

$$
v_{i}=\dot{d}_{i}=v_{0}+\omega_{0} \times\left(d_{i}-d_{0}\right)+\sum_{k=1}^{i}\left\{k_{k} \times\left(\dot{d_{i}}-p_{k}\right) \dot{\theta}_{k}\right\},
$$




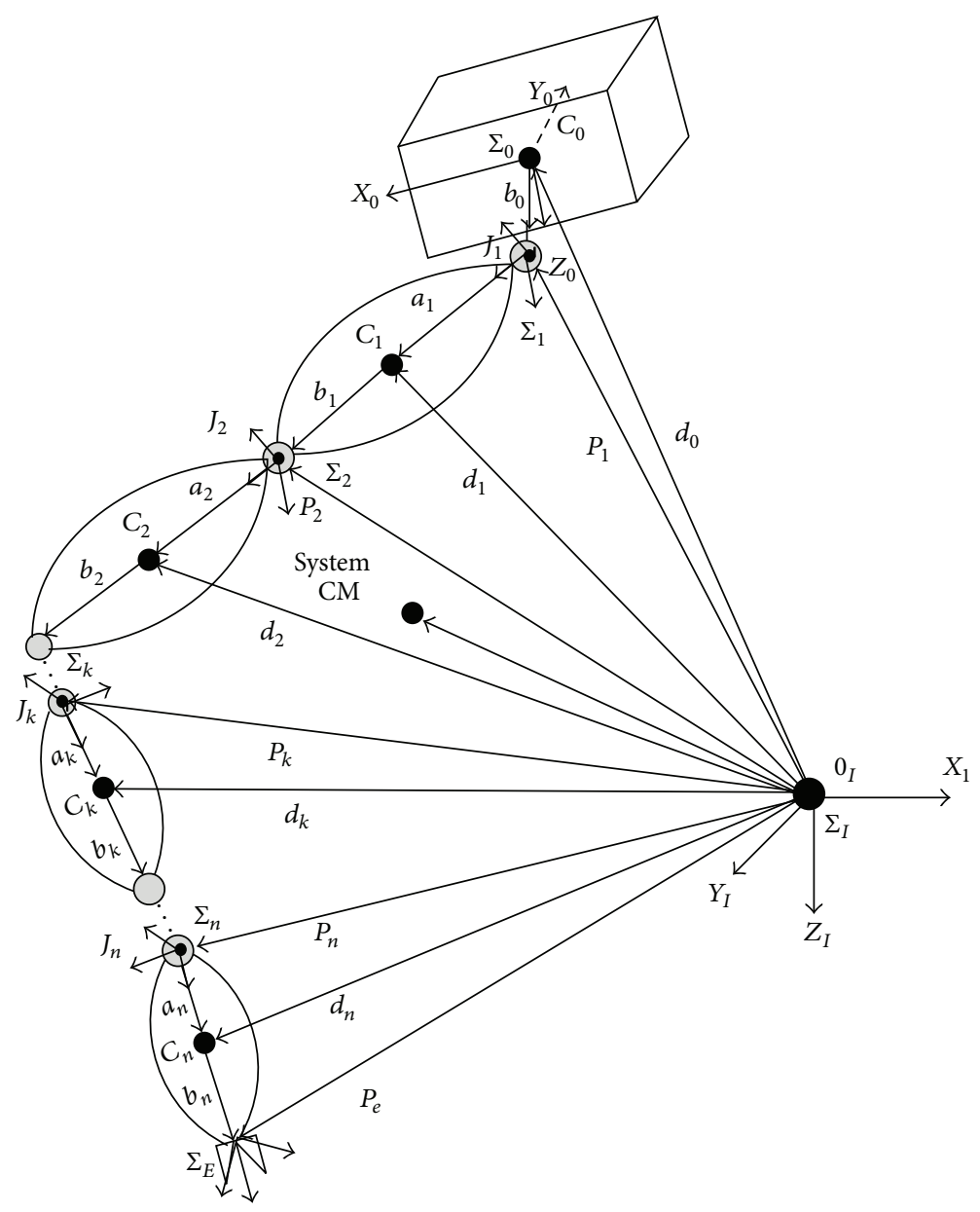

FIGURE 4: System structure of the RF-MJM system.

where $v_{0}$ and $v_{i}$ are the linear velocity of the COM of the RFR and the link $i$, respectively; $\omega_{0}$ is the angular velocity of the RFR in the frame of $\Sigma_{I} ; \theta_{k}$ is the angular position vector of the $k$ th joint; $k_{k}$ denotes the unit vector of the axis $Z_{i}$ of the $i$ th joint frame; $d_{i}, p_{k}$ represent the position vectors of the COM of the link $i$ and the $k$ th joint. The angular velocity of the $i$ th joint can be denoted as

$$
\omega_{i}=\omega_{0}+\sum_{k=1}^{i} k_{k} \dot{\theta}_{k}
$$

Combine (2) and (3), and the kinematic model of the RFMJM system is

$$
\left[\begin{array}{c}
v_{i} \\
\omega_{i}
\end{array}\right]=\bar{J}_{b i}\left[\begin{array}{c}
v_{0} \\
\omega_{0}
\end{array}\right]+\bar{J}_{m i} \dot{\Theta}
$$

where $\bar{J}_{b i}$ is the Jacobian matrix of the RFR system and has the following form:

$$
\bar{J}_{b i}=\left[\begin{array}{cc}
E & -\widetilde{d}_{0 i} \\
0 & E
\end{array}\right]+\left[\begin{array}{c}
\bar{J}_{b v_{i}} \\
\bar{J}_{b \omega_{i}}
\end{array}\right]
$$

In (5), $E$ is the unity matrix with proper dimension:

$$
d_{0 i}=d_{i}-d_{0}=\left[\begin{array}{lll}
d_{0 i, x} & d_{0 i, y} & d_{0 i, z}
\end{array}\right]^{T}
$$

and $\widetilde{d}_{0 i}$ is the skew-symmetric matrix of the vector $d_{0 i}$; that is,

$$
\widetilde{d}_{0 i}=\left[\begin{array}{ccc}
0 & -d_{0 i, z} & d_{0 i, y} \\
d_{0 i, z} & 0 & -d_{0 i, x} \\
-d_{0 i, y} & d_{0 i, x} & 0
\end{array}\right]
$$

$\bar{J}_{m i}$ in (4) is the Jacobian matrix of the manipulator system defined as

$$
\bar{J}_{m i}=\left[\begin{array}{ccc}
k_{1} \times\left(d_{i}-p_{1}\right) & \cdots & k_{i} \times\left(d_{i}-p_{i}\right) \\
k_{1} & \cdots & k_{i}
\end{array}\right]=\left[\begin{array}{c}
\bar{J}_{m v_{i}} \\
\bar{J}_{m \omega_{i}}
\end{array}\right] .
$$

Also, we can transform the linear velocity of the RFR $v_{0}$ into the velocity in the RFR body frame; that is,

$$
v_{0}=\left[\begin{array}{ccc}
c \theta c \varphi & s \phi s \theta c \varphi-c \phi s \varphi & c \phi s \theta c \varphi+s \phi s \varphi \\
c \theta s \varphi & c \phi c \varphi+s \theta s \phi s \varphi & c \phi s \theta s \varphi-s \phi c \varphi \\
-s \theta & s \phi c \theta & c \phi c \theta
\end{array}\right]\left[\begin{array}{c}
u \\
v \\
w
\end{array}\right],
$$


where $c$ and $s$ mean trigonometric function $\cos$ and $\sin$, respectively; $\Phi=[\phi, \theta, \psi]^{T}$ represents the attitude of the RFR; $[u, v, w]$ is the linear velocity of the RFR in the RFR body fixed reference frame.

Similarly, the relation between the angular velocity in the frame of $\Sigma_{I}$ and that in the body frame is as follows:

$$
\omega_{0}=\left[\begin{array}{ccc}
1 & \sin \phi \tan \theta & \cos \phi \tan \theta \\
0 & \cos \phi & -\sin \phi \\
0 & \sin \phi \sec \theta & \cos \phi \sec \theta
\end{array}\right]\left[\begin{array}{l}
p \\
q \\
r
\end{array}\right]
$$

where $p, q$, and $r$ are the components of the angular velocity along the axes of the RFR body fixed reference frame.

2.2. Dynamics Model. In this section, the dynamics model of the RF-MJM system will be deduced using Euler-Lagrange method.

2.2.1. Kinetic Energy. Firstly, the kinetic energy of the system can be denoted as

$$
E_{k}=\frac{1}{2} \sum_{i=1}^{n}\left(\omega_{i}^{T} I_{i} \omega_{i}+m_{i} v_{i}^{T} v_{i}\right)
$$

where $m_{i}$ and $I_{i}$ are the mass and the inertia tensor of the $i$ th part[10].

Substitute (2) and (3) into (11), we have

$$
E_{k}=\frac{1}{2}\left[\begin{array}{c}
v_{0} \\
\omega_{0} \\
\dot{\Theta}
\end{array}\right]^{T}\left[\begin{array}{ccc}
\omega E & \omega \widetilde{d}_{0 g}^{T} & J_{T \omega} \\
\omega \widetilde{d}_{0 g} & H_{\omega} & H_{\omega \phi} \\
J_{T \omega}^{T} & H_{\omega \phi}^{T} & H_{m}
\end{array}\right]\left[\begin{array}{c}
v_{0} \\
\omega_{0} \\
\dot{\Theta}
\end{array}\right]=\frac{1}{2}\left[\begin{array}{c}
v_{0} \\
\omega_{0} \\
\dot{\Theta}
\end{array}\right]^{T} H\left[\begin{array}{c}
v_{0} \\
\omega_{0} \\
\dot{\Theta}
\end{array}\right],
$$

where $H$ is called the inertia matrix of the RF-MJM system with

$$
\begin{gathered}
H_{m}=\sum_{i=1}^{n}\left(J_{R i}^{T} I J_{R i}+m_{i} J_{T i}^{T} J_{T i}\right) \\
J_{T \omega}=\sum_{i=1}^{n}\left(m_{i} J_{T i}\right) \\
H_{\omega}=\sum_{i=1}^{n}\left(I_{i}+m_{i} \widetilde{d}_{0 i}^{T} \tilde{d}_{0 i}\right)+I_{0} \\
H_{\omega \phi}=\sum_{i=1}^{n}\left(I_{i} J_{R i}+m_{i} \widetilde{d}_{0 i}^{T} J_{T i}\right)
\end{gathered}
$$

$$
J_{R i}=\left[\begin{array}{lllllll}
k_{1} & k_{2} & \cdots & k_{i} & 0 & \cdots & 0
\end{array}\right]
$$

$J_{T i}$

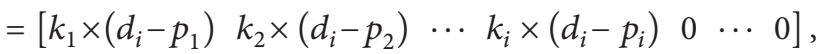

$$
\begin{aligned}
& d_{g}=\frac{\sum_{1=0}^{n} m_{i} d_{i}}{\sum_{1=0}^{n} m_{i}} .
\end{aligned}
$$

2.2.2. Potential Energy. In this part, the potential energy due to the gravity will be deduced. Firstly, based on Figure 2, (1) can be rewritten as

$$
d_{i}=d_{0}+\sum_{k=1}^{i}\left({ }^{I} A_{k-1} C_{k-1, k}-{ }^{I} A_{k} C_{k, k}\right),
$$

where ${ }^{I} A_{0}$ and ${ }^{I} A_{k}$ denote the coordinate transformation matrix from the frame $\Sigma_{0}$ and the frame $\Sigma_{k}$ to the frame $\Sigma_{I}$, respectively; $C_{k, k}$ is the position vector from joint $i$ to the COM of the $i$ th partdenoted in the $i$ th joint coordinate system.

With (14), the potential energy due to gravity of the RFMJM system can be easily obtained as follows:

$$
\begin{aligned}
E_{p} & =-\sum_{i=0}^{n} m_{i} G^{T} d_{i} \\
& =\sum_{i=0}^{n} m_{i} G^{T}\left(d_{0}+\sum_{k=1}^{i-1}\left({ }^{I} A_{k-1} C_{k-1, k}-{ }^{I} A_{k-1} C_{k, k}\right)\right),
\end{aligned}
$$

where

$$
G=\left[\begin{array}{l}
0 \\
0 \\
g
\end{array}\right]
$$

and $g$ is the acceleration due to gravity.

2.2.3. Dynamics Model. The Euler-Lagrangian equation of the RF-MJM system is

$$
L=E_{k}-E_{p}
$$

From (12), the kinetic energy can be rewritten as

$$
\begin{aligned}
E_{k}=\frac{1}{2}[\omega & v_{0}^{T} E v_{0}+\Phi \omega_{0}^{T} \widetilde{d}_{0 g} v_{0}+\dot{\Theta}^{T} J_{T \omega}^{T} v_{0} \\
& +\Phi v_{0}^{T} \tilde{d}_{0 g}^{T} \omega_{0}+\omega_{0}^{T} H_{\omega} \omega_{0}+\dot{\Theta}^{T} H_{\omega \phi}^{T} \omega_{0} \\
& \left.+v_{0}^{T} J_{T \omega} \dot{\Theta}+\omega_{0}^{T} H_{\omega \phi} \dot{\Theta}+\dot{\Theta}^{T} H_{m} \dot{\Theta}\right] .
\end{aligned}
$$


Thus we have

$$
\begin{gathered}
\frac{\partial E_{k}}{\partial \dot{q}}=\left[\begin{array}{l}
\frac{\partial E_{k}}{\partial v_{0}} \\
\frac{\partial E_{k}}{\partial \omega_{0}} \\
\frac{\partial E_{k}}{\partial \dot{\Theta}}
\end{array}\right]=\left[\begin{array}{c}
\omega E v_{0}+\omega \widetilde{d}_{0 g}^{T} \omega_{0}+J_{T \omega} \dot{\Theta} \\
H_{\omega} \omega_{0}+\omega \widetilde{d}_{0 g} v_{0}+H_{\omega \phi} \dot{\Theta} \\
H_{m} \dot{\Theta}+H_{\omega \phi}^{T} \omega_{0}+J_{T \omega}^{T} v_{0}
\end{array}\right] \\
\frac{d}{d t}\left(\frac{\partial E_{k}}{\partial \dot{q}}\right)=\left[\begin{array}{c}
\frac{d}{d t}\left(\frac{\partial E_{k}}{\partial v_{0}}\right) \\
\frac{d}{d t}\left(\frac{\partial E_{k}}{\partial \omega_{0}}\right) \\
\frac{d}{d t}\left(\frac{\partial E_{k}}{\partial \dot{\Theta}}\right)
\end{array}\right]=\left[\begin{array}{c}
\omega E \dot{v}_{0}+\omega \dot{\tilde{d}}_{0 g}^{T} \omega_{0}+\omega \widetilde{d}_{0 g}^{T} \dot{\omega}_{0}+J_{T \omega} \ddot{\Theta}+\dot{J}_{T \omega} \dot{\Theta} \\
\dot{H}_{\omega} \omega_{0}+H_{\omega} \dot{\omega}_{0}+\omega \dot{\tilde{d}}_{0 g} v_{0}+\omega \tilde{d}_{0 g} \dot{v}_{0}+\dot{H}_{\omega \phi} \dot{\Theta}+H_{\omega \phi} \ddot{\Theta} \\
\dot{H}_{m} \dot{\Theta}+H_{m} \ddot{\Theta}+\dot{H}_{\omega \phi}^{T} \omega_{0}+H_{\omega \phi}^{T} \dot{\omega}_{0}+\dot{J}_{T \omega}^{T} v_{0}+J_{T \omega}^{T} \dot{v}_{0}
\end{array}\right] \\
\frac{\partial E_{k}}{\partial q}=\left[\begin{array}{l}
\frac{\partial E_{a}}{\partial p}+\frac{\partial E_{b}}{\partial p}+\frac{\partial E_{c}}{\partial p} \\
\frac{\partial E_{a}}{\partial \Phi}+\frac{\partial E_{b}}{\partial \Phi}+\frac{\partial E_{c}}{\partial \Phi} \\
\frac{\partial E_{a}}{\partial \Theta}+\frac{\partial E_{b}}{\partial \Theta}+\frac{\partial E_{c}}{\partial \Theta}
\end{array}\right]
\end{gathered}
$$

where

$$
q=\left[\begin{array}{l}
X \\
\Phi \\
\Theta
\end{array}\right], \quad \dot{q}=\left[\begin{array}{c}
v_{0} \\
\omega_{0} \\
\dot{\Theta}
\end{array}\right]
$$

are the position vector and the velocity vector of the RF-MJM system, respectively; $X$ is the position vector of the RFR; and

$$
\begin{aligned}
& \frac{\partial E_{p}}{\partial \Phi} \\
& =-\left[\begin{array}{c}
\frac{\partial E_{p}}{\partial \phi} \\
\frac{\partial E_{p}}{\partial \theta} \\
\frac{\partial E_{p}}{\partial \varphi}
\end{array}\right]
\end{aligned}
$$

$$
\begin{aligned}
& E_{a}=\omega \omega_{0}^{T} \tilde{d}_{0 g} v_{0} ; \quad E_{b}=\frac{1}{2} \omega_{0}^{T} H_{\omega} \omega_{0} \\
& E_{c}=\left(v_{0}^{T} J_{T \omega}+\omega_{0}^{T} H_{\omega \phi}+\frac{1}{2} \dot{\Theta}^{T} H_{m}\right) \dot{\Theta} .
\end{aligned}
$$

Similarly, with respect to the potential energy term, we have

$$
\frac{\partial E_{p}}{\partial X}=\sum_{i=0}^{n} m_{i}\left[\begin{array}{c}
\frac{\partial g^{T} d_{0}}{\partial x_{0}} \\
\frac{\partial g^{T} d_{0}}{\partial y_{0}} \\
\frac{\partial g^{T} d_{0}}{\partial z_{0}}
\end{array}\right]
$$

$$
\begin{aligned}
\frac{\partial E_{p}}{\partial \Theta}=-\left(\sum_{i=0}^{n} m_{i} \partial g^{T}\right. & \\
& \left.\times\left(d_{0}+\sum_{j=1}^{i}\left({ }^{I} A_{j-1} * C_{j-1, j}-{ }^{I} A_{j} * C_{j, j}\right)\right)\right)
\end{aligned}
$$$$
\times(\partial \Theta)^{-1}
$$

$$
=-\left[\begin{array}{c}
\frac{-\sum_{i=1}^{n} m_{i} \partial g{ }^{T} \sum_{j=1}^{i}\left({ }^{I} A_{j-1} * C_{j-1, j}-{ }^{I} A_{j} * C_{j, j}\right)}{\partial \phi} \\
\frac{-\sum_{i=1}^{n} m_{i} \partial g^{T} \sum_{j=1}^{i}\left({ }^{I} A_{j-1} * C_{j-1, j}-{ }^{I} A_{j} * C_{j, j}\right)}{\partial \theta} \\
-\sum_{i=1}^{n} m_{i} \partial g \sum^{T} \sum_{j=1}^{i}\left({ }^{I} A_{j-1} * C_{j-1, j}-{ }^{I} A_{j} * C_{j, j}\right) \\
\partial \varphi
\end{array}\right] .
$$

Thus, the dynamics model of the RF-MJM system can be obtained through the following Euler-Lagrange equation:

$$
\frac{d}{d t} \frac{\partial E_{k}}{\partial \dot{q}}-\frac{\partial E_{k}}{\partial q}+\frac{\partial E_{P}}{\partial q}=\tau \text {. }
$$

Substitute (20)-(21) and (24)-(26) into (27) and after simplifying we can obtain the following dynamics model of the RF-MJM system:

$$
\left[\begin{array}{cc}
H_{b} & H_{b m} \\
H_{b m}^{T} & H_{m}
\end{array}\right]\left[\begin{array}{c}
\ddot{X}_{b} \\
\ddot{\Theta}
\end{array}\right]+\left[\begin{array}{c}
C_{b} \\
C_{m}
\end{array}\right]+\left[\begin{array}{c}
G_{b} \\
G_{m}
\end{array}\right]=\left[\begin{array}{c}
F_{p} \\
\tau_{m}
\end{array}\right],
$$

where

$$
\ddot{X}_{b}=\left[\begin{array}{c}
\dot{v}_{0} \\
\dot{\omega}_{0}
\end{array}\right] .
$$


$C_{b}$ and $C_{m}$ are the Coriolis and centrifugal force of the system; $G_{b}$ and $G_{m}$ are the force due to gravity;

$$
F_{p}=\left[\begin{array}{c}
F_{B} \\
M_{B}
\end{array}\right] ; \quad F_{B}=\left[\begin{array}{c}
F_{x} \\
F_{y} \\
F_{z}
\end{array}\right] ; \quad M_{B}=\left[\begin{array}{c}
L \\
M \\
N
\end{array}\right]
$$

are the force and moment produced by the RFR; the other terms are defined as follows:

$$
\begin{aligned}
& H_{b}=\left[\begin{array}{ll}
H_{b_{11}} & H_{b_{12}} \\
H_{b_{21}} & H_{b_{22}}
\end{array}\right] \\
& H_{b_{11}}=\omega E ; \quad H_{b_{12}}=\omega \tilde{d}_{o g}^{T} \\
& H_{b_{21}}=\omega \tilde{d}_{o g} ; \quad H_{b_{22}}=H_{\omega} \\
& H_{b m}=\left[\begin{array}{l}
H_{b m v} \\
H_{b m \omega}
\end{array}\right]_{2 \times 1} \\
& H_{b_{m v}}=J_{T \omega} ; \quad H_{b m \omega}=H_{\omega \phi} \\
& C_{b}=\left[\begin{array}{l}
C_{b v} \\
C_{b \omega}
\end{array}\right] \\
& C_{b v}=\omega \dot{\vec{d}}_{0 g}^{T} \omega_{0}+\dot{J}_{T \omega} \dot{\Theta}-\omega \omega_{0}^{T} \frac{\partial \widetilde{d}_{0 g}}{\partial X} v_{0}-v_{0}^{T} \frac{\partial J_{T \omega}}{\partial X} \dot{\Theta} \\
& -\omega_{0}^{T} \frac{\partial H_{\omega \phi}}{\partial X} \dot{\Theta}-\frac{1}{2}\left(\omega_{0}^{T} \frac{\partial H_{\omega}}{\partial X} \omega_{0}+\dot{\Theta}^{T} \frac{\partial H_{m}}{\partial X} \dot{\Theta}\right) \\
& C_{b \omega}=\dot{H}_{\omega} \omega_{0}+\omega \dot{\vec{d}}_{0 g} v_{0}+\dot{H}_{\omega \phi} \dot{\Theta}-\omega \omega_{0}^{T} \frac{\partial \widetilde{d}_{0 g}}{\partial \Phi} v_{0}-v_{0}^{T} \frac{\partial J_{T \omega}}{\partial \Phi} \dot{\Theta} \\
& -\omega_{0}^{T} \frac{\partial H_{\omega \phi}}{\partial \Phi} \dot{\Theta}-\frac{1}{2}\left(\omega_{0}^{T} \frac{\partial H_{\omega}}{\partial \Phi} \omega_{0}+\dot{\Theta}^{T} \frac{\partial H_{m}}{\partial \Phi} \dot{\Theta}\right) . \\
& C_{m}=\frac{1}{2} \dot{J}_{T \omega}^{T} v_{0}+\frac{1}{2} v_{0}^{T} \dot{J}_{T \omega}+\frac{1}{2} \dot{H}_{\omega \phi}^{T} \omega_{0}+\frac{1}{2} \omega_{0}^{T} \dot{H}_{\omega \phi}+\dot{H}_{m} \dot{\Theta} .
\end{aligned}
$$

2.2.4. Extended Dynamics Model. When the manipulator contacts some external objects, the dynamics model (28) becomes

$$
\left[\begin{array}{cc}
H_{b} & H_{b m} \\
H_{b m}^{T} & H_{m}
\end{array}\right]\left[\begin{array}{c}
\ddot{X}_{b} \\
\ddot{\Theta}
\end{array}\right]+\left[\begin{array}{c}
C_{b} \\
C_{m}
\end{array}\right]+\left[\begin{array}{c}
G_{b} \\
G_{b}
\end{array}\right]=\left[\begin{array}{c}
F_{p} \\
\tau_{m}
\end{array}\right]+\left[\begin{array}{c}
J_{b}^{T} \\
J_{m}^{T}
\end{array}\right] F_{e},
$$

where $F_{e}$ is the force and torque exerting on the end of the manipulator; $J_{b}$ and $J_{m}$ are the Jacobian matrix defined as

$$
\begin{gathered}
J_{b}=\left[\begin{array}{cc}
E & -\tilde{p}_{0 i} \\
0 & E
\end{array}\right] ; \quad p_{0 i}=p_{i}-d_{0} ; \\
J_{m}=\left[\begin{array}{rrrr}
k_{1} \times\left(p_{e}-p_{1}\right) & k_{2} \times\left(p_{e}-p_{2}\right) & \cdots & k_{n} \times\left(p_{e}-p_{n}\right) \\
k_{1} & k_{2} & \cdots & k_{n}
\end{array}\right] .
\end{gathered}
$$

Furthermore, if we consider the aerodynamics of the RFR system, the force and moment produced by the RFR $F_{p}$ can be denoted as the following mathematical equations [11]:

$$
\begin{gathered}
F_{X}=-T_{M} \sin a_{1 s} ; \quad F_{Y}=T_{M} \sin b_{1 s}-T_{T} ; \\
F_{Z}=-T_{M} \cos a_{1 s} \cos b_{1 s} ; \\
L=-\left(\frac{\partial L_{M}}{\partial b_{1 s}}\right) b_{1 s}-Q_{M} \sin a_{1 s} \\
M=\left(\frac{\partial M_{M}}{\partial a_{1 s}}\right) a_{1 s}-Q_{M} \sin b_{1 s}-Q_{T} \\
N=-Q_{M} \cos a_{1 s} \cos b_{1 s}
\end{gathered}
$$

where $T_{M}$ and $T_{T}$ are the forces derived from the main rotor and tail rotor of the RFR, and $a_{1 s}$ and $b_{1 s}$ stand for the longitudinal and lateral flapping angle of main rotor, respectively; the forces $T_{M}$ and $T_{T}$ and the moments $Q_{M}$ and $Q_{T}$ can be calculated as $[12,13]$.

Up to now, we have constructed the nonlinear dynamics model of the RF-MJM system.

\section{Analysis of Dynamics Model and Linearization}

3.1. Analysis of the Dynamics Model. In order to understand the coupling between the RFR and manipulator clearly, we rewrite the system model (28) as follows:

$$
\begin{gathered}
{\left[\begin{array}{cc}
H_{b}(\Phi, \Theta) & H_{b m}(\Phi, \Theta) \\
H_{b m}^{T}(\Phi, \Theta) & H_{m}(\Phi, \Theta)
\end{array}\right]\left[\begin{array}{c}
\ddot{X}_{b} \\
\ddot{\Theta}
\end{array}\right]+\left[\begin{array}{c}
C_{b}\left(v_{0}, \omega_{0}, \Theta, \Phi, \dot{\Phi}\right) \\
C_{m}\left(v_{0}, \omega_{0}, \Theta, \Phi, \dot{\Phi}\right)
\end{array}\right]} \\
+\left[\begin{array}{c}
G_{b}(X, \Theta, \Phi) \\
G_{b}(\Theta, \Phi)
\end{array}\right]=\left[\begin{array}{c}
F_{p} \\
\tau_{m}
\end{array}\right] .
\end{gathered}
$$

From (35), it can be easily seen that the coupling between the RFR and the manipulator appears in all the terms except for the exerting force/moment. That means the RF-MJM system model is more complicated than the RFR system model as shown in [11]. These can be summarized as follows.

(1) Compared to the RFR model, there are some new terms in the system (28), such as $C_{b v}$. These new terms make the system model more complicated, and the result is that some control algorithm that has been shown to be fit for RFR system cannot be used directly in the RF-MJM system. For example, in reference [11], a RFR system is proved to be approximate feedback linearizable. This, unfortunately, cannot be implemented in the RF-MJM system.

(2) The system structure of the RF-MJM is of great complication compared to the RFR system. This can be easily seen through the preceding system equations. Again, the reason is because of the coupling between the RFR and the manipulator. The higher 
complication results in heavier nonlinearity which makes the controller design of the RF-MJM systemextraordinarily challenging.

3.2. Linearization and $L Q R$ Controller Design. In the above section, we have obtained the detailed mathematical model of the RF-MJM system, which can be easily computed and simulated through using symbolic computation toolbox of MATLAB. However, this kind of controller is difficult to be used due to the high complexity and nonlinearities. Thus, in this section, we will try to find the linearized model of the RFMJM system and analyze the influence of the parameters on the system parameters.

The linearization can be implemented through the following steps: firstly, search the trim point of the RF-MJM system; secondly, compute the derivatives of the system model with respect to the state and input to obtain the system matrix of the desired linear model. With the linearized model, some linear controller design strategies, for example, the LQR controller, can be used to stabilize the original nonlinear system. In the following content of this section, taking one-joint RF-MJM system as an example, the linearization and the LQR control design will be conducted and system performance will be analyzed.

A trim point, also known as an equilibrium point, of a nonlinear system is a point in the state space of a dynamic system, and at this point, the derivatives of the states with respect to time are precisely zeros.

The state vector of the RF-MJM can be denoted as

$$
\left[\begin{array}{llllllllllllll}
x & y & z & \phi & \theta & \psi & u & v & w & p & q & r & \Theta & \Theta
\end{array}\right]
$$

and the input vector is

$$
\left[\begin{array}{lllll}
a_{1 s} & b_{1 s} & \theta_{M} & \theta_{T} & \tau_{\Theta}
\end{array}\right]
$$

where $a_{1 s}$ and $b_{1 s}$ are the cyclic pitch angle of the main rotor, respectively; $\theta_{M}$ and $\theta_{T}$ are the collective pitch angle of the main rotor and the tilt rotor, respectively.

Based on the definition of trim point, all the velocity state should be set to zeros; that is,

$$
u=v=w=p=q=r=\dot{\Theta}=0 .
$$

Also, all the derivatives of the states with respect to time should be zeros. Under condition (38), this is equivalent to

$$
\left[\begin{array}{c}
\ddot{X}_{b} \\
\ddot{\Theta}
\end{array}\right]=H^{-1}\left(\left[\begin{array}{c}
F_{p} \\
\tau_{m}
\end{array}\right]+\left[\begin{array}{c}
J_{b}^{T} \\
J_{m}^{T}
\end{array}\right] F_{e}-\left[\begin{array}{c}
C_{b} \\
C_{m}
\end{array}\right]-\left[\begin{array}{c}
G_{b} \\
G_{b}
\end{array}\right]\right)=0_{7 \times 1} \text {. }
$$

The right-hand side of (31) is only related to $\phi, \theta, \psi, \Theta$, and input vector, so we have 9 free variables and 7 equalities. Furthermore, if we define $\tau_{\Theta}=0$ and $\psi=0$, we will have only 7 free variables. Thus, the trim point can be obtained directly by solving the nonlinear equalities (39), which can be easily conducted using some searching function in MATLAB.

In order to evaluate the influence of the mass of the manipulator on the whole system, we list out the trim point
TABle 1: Parameters of RF-MJM system.

\begin{tabular}{lcc}
\hline Parameter & Describe & Unit \\
\hline$m_{0}=9.5$ & The mass of rotor-flying robot (RFR) & $\mathrm{kg}$ \\
$m_{1}=2.5$ & The mass of manipulator & $\mathrm{kg}$ \\
$I_{0 x x}=0.1634$ & Moment of inertia of RFR & $\mathrm{kgm}^{2}$ \\
$I_{0 y y}=0.5782$ & Moment of inertia of RFR & $\mathrm{kgm}^{2}$ \\
$I_{0 z z}=0.6306$ & Moment of inertia of RFR & $\mathrm{kgm}^{2}$ \\
$I_{1 x x}=0.1399$ & Moment of inertia of manipulator & $\mathrm{kgm}^{2}$ \\
$I_{1 y y}=0.1399$ & Moment of inertia of manipulator & $\mathrm{kgm}^{2}$ \\
$I_{1 z z}=0.00112$ & Moment of inertia of manipulator & $\mathrm{kgm}^{2}$ \\
$l_{0}=0.3$ & The length from the centroid of RFR & $\mathrm{m}$ \\
$l_{1}=0.4$ & The length of half of the first link & $\mathrm{m}$ \\
$\phi=0.0769$ & Roll angle & $\mathrm{rad}$ \\
$\theta=0.0211$ & Pitch angle & $\mathrm{rad}$ \\
$\psi=$ any value & Yaw angle & $\mathrm{rad}$ \\
$\theta_{1}=0.0211$ & Joint movement angle & $\mathrm{rad}$ \\
$F_{0}=\left[\begin{array}{lll}0 & 0 & 0\end{array}\right]^{T}$ & External force & $\mathrm{N}$ \\
$T_{0}=\left[\begin{array}{lll}0 & 0 & 0\end{array}\right]^{T}$ & External torque & $\mathrm{Nm}$ \\
\hline
\end{tabular}

of the linearization system with different manipulator masses (the parameters of the system are listed out in Table 1, and the trim point is in Table 2 in the next section).

The linearization system model is

$$
\Delta \dot{X}=A \Delta X+B \Delta u
$$

where

$$
\begin{gathered}
\Delta X=X-X_{\text {trim }} \\
A=\left[\begin{array}{cccccc}
0_{3 \times 3} & E_{3} & 0_{3 \times 3} & 0_{3 \times 3} & 0_{3 \times 1} & 0_{3 \times 1} \\
A_{1} & A_{2} & A_{3} & A_{4} & A_{5} & A_{6} \\
0_{3 \times 3} & 0_{3 \times 3} & 0_{3 \times 3} & E & 0_{3 \times 1} & 0_{3 \times 1} \\
A_{7} & A_{8} & A_{9} & A_{10} & A_{11} & A_{12} \\
0_{1 \times 3} & 0_{1 \times 3} & 0_{1 \times 3} & 0_{1 \times 3} & 0 & 1 \\
A_{13} & A_{14} & A_{15} & A_{16} & A_{17} & A_{18}
\end{array}\right] \\
B=\left[\begin{array}{llllll}
0_{5 \times 3} & B_{1}^{T} & 0_{5 \times 3} & B_{2}^{T} & 0_{5 \times 1} & B_{3}^{T}
\end{array}\right]^{T} .
\end{gathered}
$$

Furthermore, in order to analyze the performance of system (40), the eigenvalues of $A$ matrix are given in Figure 5. From it, we can get the following results.

(1) The whole system is static-instable since it has positive eigenvalues.

(2) With increase of the manipulator mass, the distribution of eigenvalues will be more diverging.

3.3. LQR Controller Design. Next, we will design the full state-feedback linear quadratic regulation (LQR) controller for the RFM system. In the state-feedback version of the LQR problem [14], we assume that the whole state $x$ can be measured and therefore it is available to control. Solution to the optimal state-feedback LQR problem is to find $u(t)=$ $-K x(t)$ that minimizes

$$
J_{\mathrm{LQR}}=\int_{0}^{\infty}\left(x^{T} \mathrm{Q} x+u^{T} R u\right) d t
$$




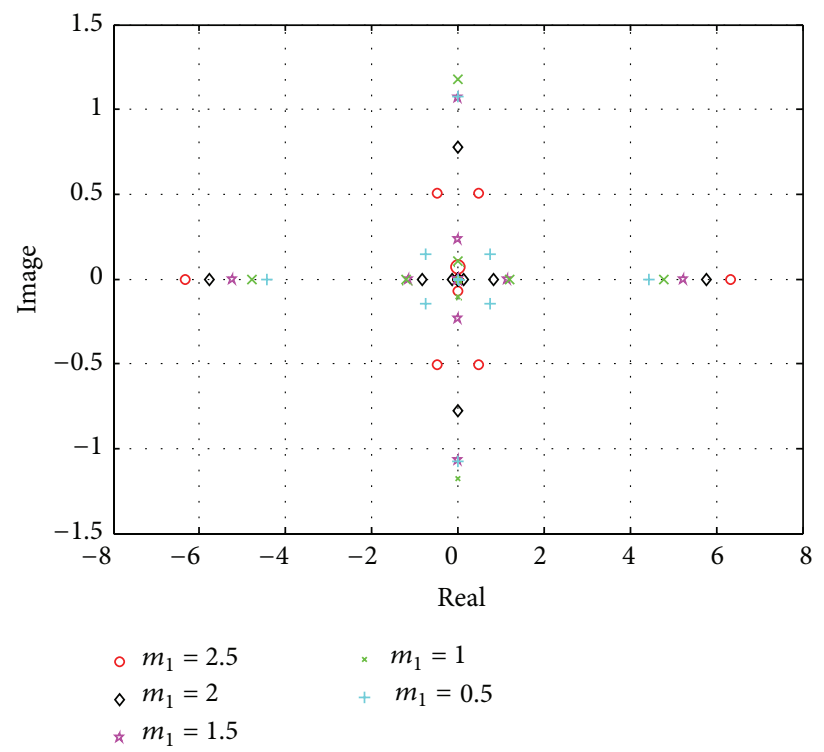

FIGURE 5: Eigenvalue distribution of $A$ matrix with different manipulator masses.

TABLE 2: Trim points corresponding to different masses of manipulator.

\begin{tabular}{lccccc}
\hline Trim point & $m_{1}=2.5 \mathrm{~kg}$ & $m_{1}=2 \mathrm{~kg}$ & $m_{1}=1.5 \mathrm{~kg}$ & $m_{1}=1 \mathrm{~kg}$ & 0.0662 \\
\hline$\phi$ & 0.0769 & 0.0732 & 0.0697 & 0.0190 & 0.0626 \\
$\theta$ & 0.0211 & 0.0204 & 0.0197 & 0.0191 & 0.0183 \\
$\theta_{1}$ & 0.0211 & 0.0205 & 0.0198 & 0.0354 & 0.0183 \\
$\theta_{M}$ & 0.0436 & 0.0409 & 0.0381 & -0.1169 & 0.0326 \\
$\theta_{T}$ & -0.1282 & -0.1245 & -0.1207 & 0.0190 & -0.1130 \\
$a_{1 s}$ & 0.0211 & 0.0204 & 0.0197 & 0.0123 & 0.0183 \\
$b_{1 s}$ & 0.0195 & 0.0169 & 0.0145 & 0.0103 \\
\hline
\end{tabular}

where $K$ is given by $K=R^{-1} B^{T} P$ and $P$ is found by solving some continuous time algebraic Riccati equations. So we can easily get the eigenvalues of the open loop system and the closed loop system by the matrices $A$ and $A-B K$, respectively.

\section{Simulations}

In this section, simulations will be conducted using the preceding nonlinear system model and the LQR controller. In the simulation, the manipulator's mass is $2.5 \mathrm{~kg}$, and the other parameters are given in Table 1 .

And the trim point of the whole system is listed out in Table 2.

With these parameters, the system matrices $A$ and $B$ are as follows:

$$
\begin{aligned}
A & =\left[\begin{array}{cccccc}
0_{3 \times 3} & E & 0_{3 \times 3} & 0_{3 \times 3} & 0_{3 \times 1} & 0_{3 \times 1} \\
0_{3 \times 3} & 0_{3 \times 3} & \bar{A}_{3} & 0_{3 \times 3} & \bar{A}_{5} & 0_{3 \times 1} \\
0_{3 \times 3} & 0_{3 \times 3} & 0_{3 \times 3} & E & 0_{3 \times 1} & 0_{3 \times 1} \\
0_{3 \times 3} & 0_{3 \times 3} & \bar{A}_{9} & 0_{3 \times 3} & \bar{A}_{11} & 0_{3 \times 1} \\
0_{1 \times 3} & 0_{1 \times 3} & 0_{1 \times 3} & 0_{1 \times 3} & 0 & 1 \\
0_{1 \times 3} & 0_{1 \times 3} & \bar{A}_{15} & 0_{1 \times 3} & \bar{A}_{17} & 0
\end{array}\right] \\
B & =\left[\begin{array}{llllll}
0_{14 \times 3} & \bar{B}_{1}^{T} & 0_{14 \times 3} & \bar{B}_{2}^{T} & 0_{14 \times 1} & \bar{B}_{3}^{T}
\end{array}\right]^{T},
\end{aligned}
$$

where

$$
\begin{gathered}
\bar{A}_{3}=\left[\begin{array}{ccc}
0.0015 & 9.7636 & 0.9945 \\
-9.8000 & -0.0005 & 0.2102 \\
0.0001 & 0.0005 & 0.0014
\end{array}\right] ; \\
\bar{A}_{9}=\left[\begin{array}{ccc}
-0.0087 & -0.0083 & 0.1834 \\
-0.0130 & 0.0255 & -0.8587 \\
-0.2013 & -0.2456 & -0.0635
\end{array}\right] ; \\
\bar{A}_{5}=\left[\begin{array}{ccc}
3.5861 \\
-0.0077 \\
0.0310
\end{array}\right] ; \\
\bar{A}_{15}=\left[\begin{array}{ccc}
0.0110 & -0.0924 & 2.6951
\end{array}\right] ; \\
\bar{A}_{17}=40.1487 ; \\
\bar{B}_{1}^{T}=\left[\begin{array}{ccc}
-0.0547 \\
-0.5451 & -7.9183 & -147.7390 \\
-1.2434 & -9.3478 & 0.6158 \\
-21.7522 & 0.4534 & -0.2015 \\
-0.5829 & 7.2377 & -0.7650 \\
0.2990 & -0.0006 & 0.0009
\end{array}\right] ;
\end{gathered}
$$




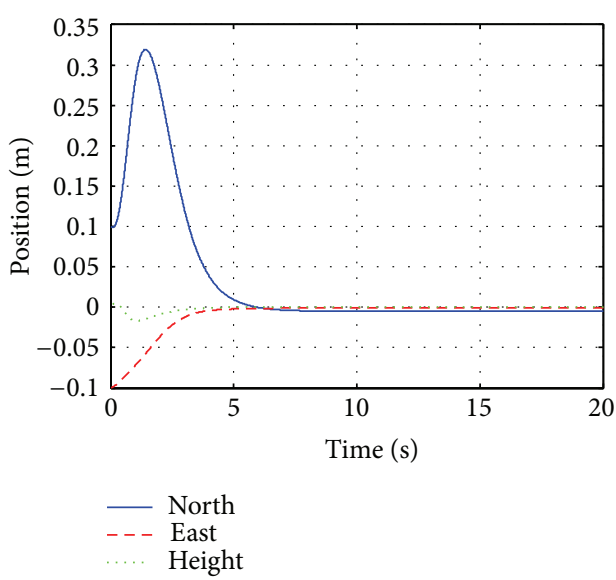

(a)

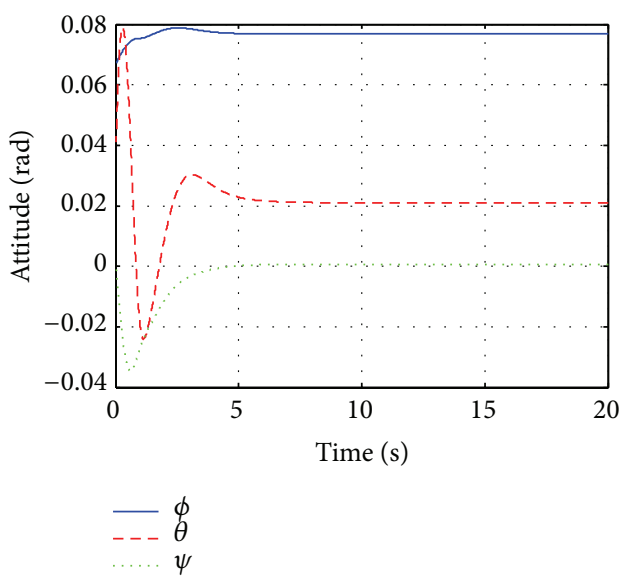

(c)

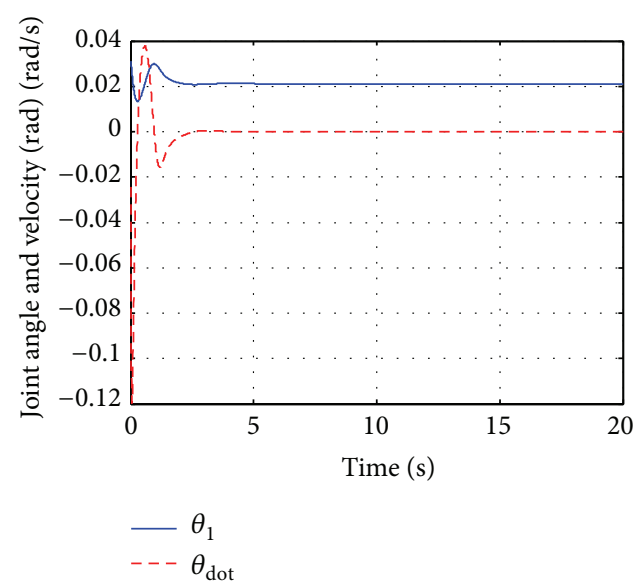

(e)

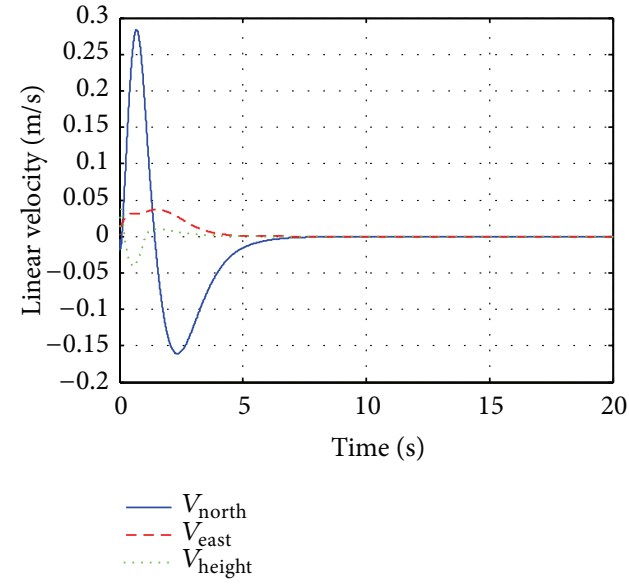

(b)

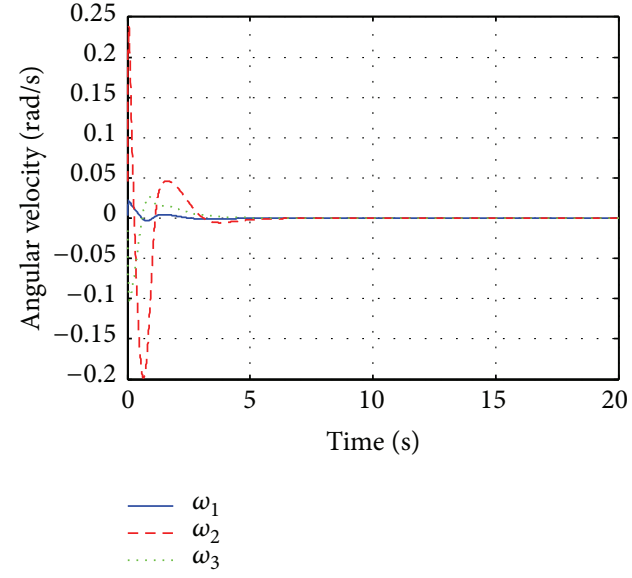

(d)
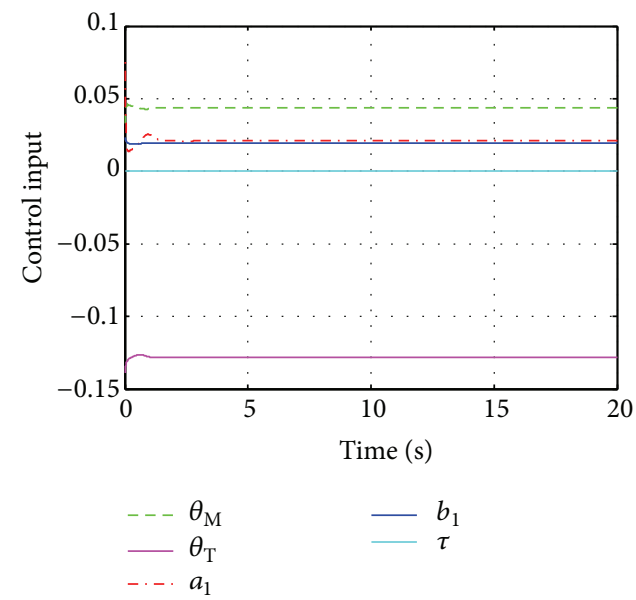

(f)

FIGURE 6: States and inputs profile under LQR controller (46).

$$
\bar{B}_{2}^{T}=\left[\begin{array}{ccc}
16.7653 & -2.5282 & 168.3611 \\
-32.8659 & 14.0449 & 125.4196 \\
20.9082 & 178.3726 & 4.0958 \\
-121.2649 & 11.0285 & -0.2925 \\
0.0011 & 0.6932 & -0.0534
\end{array}\right] \text {; }
$$

$$
\bar{B}_{3}^{T}=\left[\begin{array}{lllll}
-3.9371 & -9.0113 & -99.1817 & -4.2009 & 3.4137
\end{array}\right] .
$$

From these equations, it can be seen that the coupling between the manipulator and RFR, denoted by $\bar{A}_{5}, \bar{A}_{11}, \bar{A}_{15}$, 

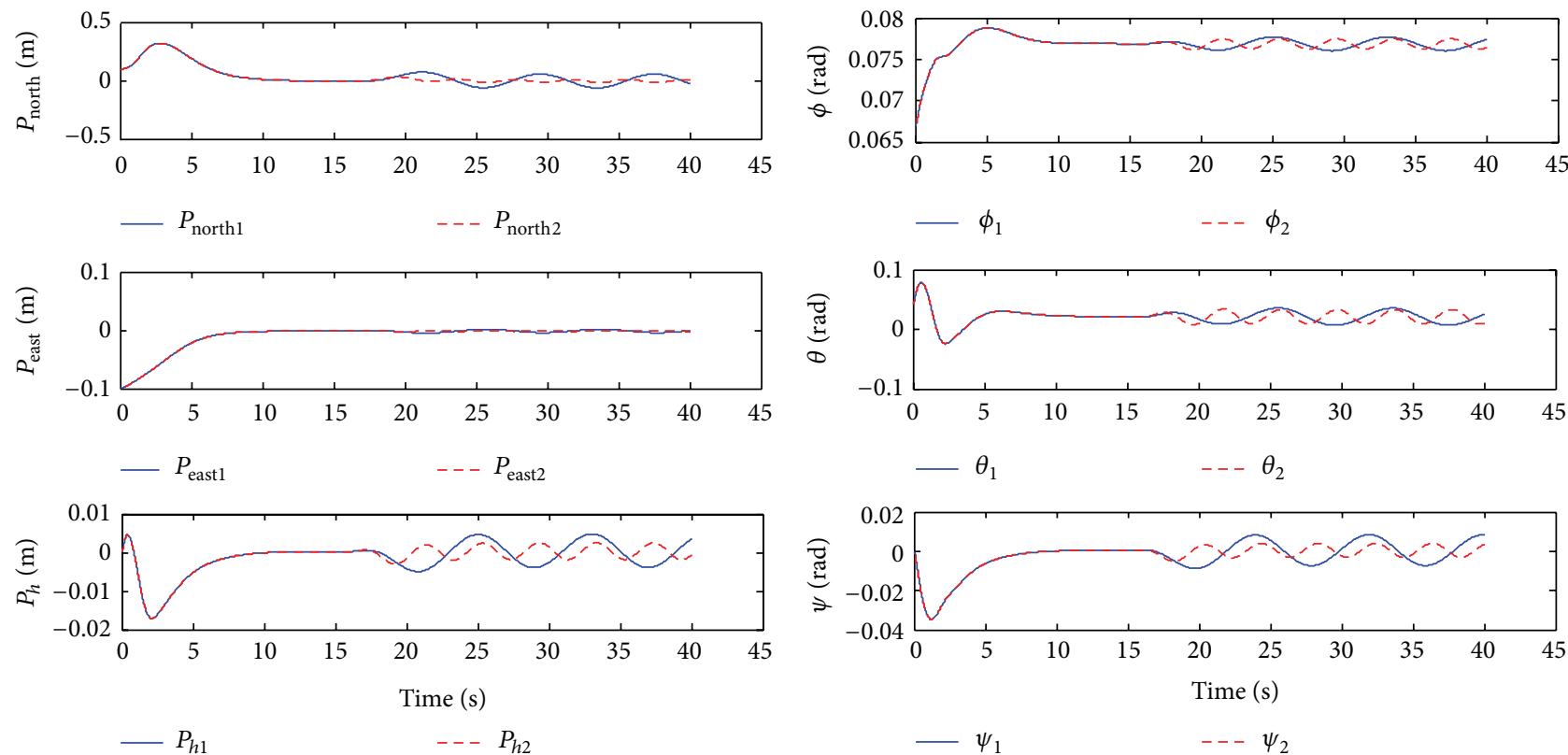

(a)

(b)
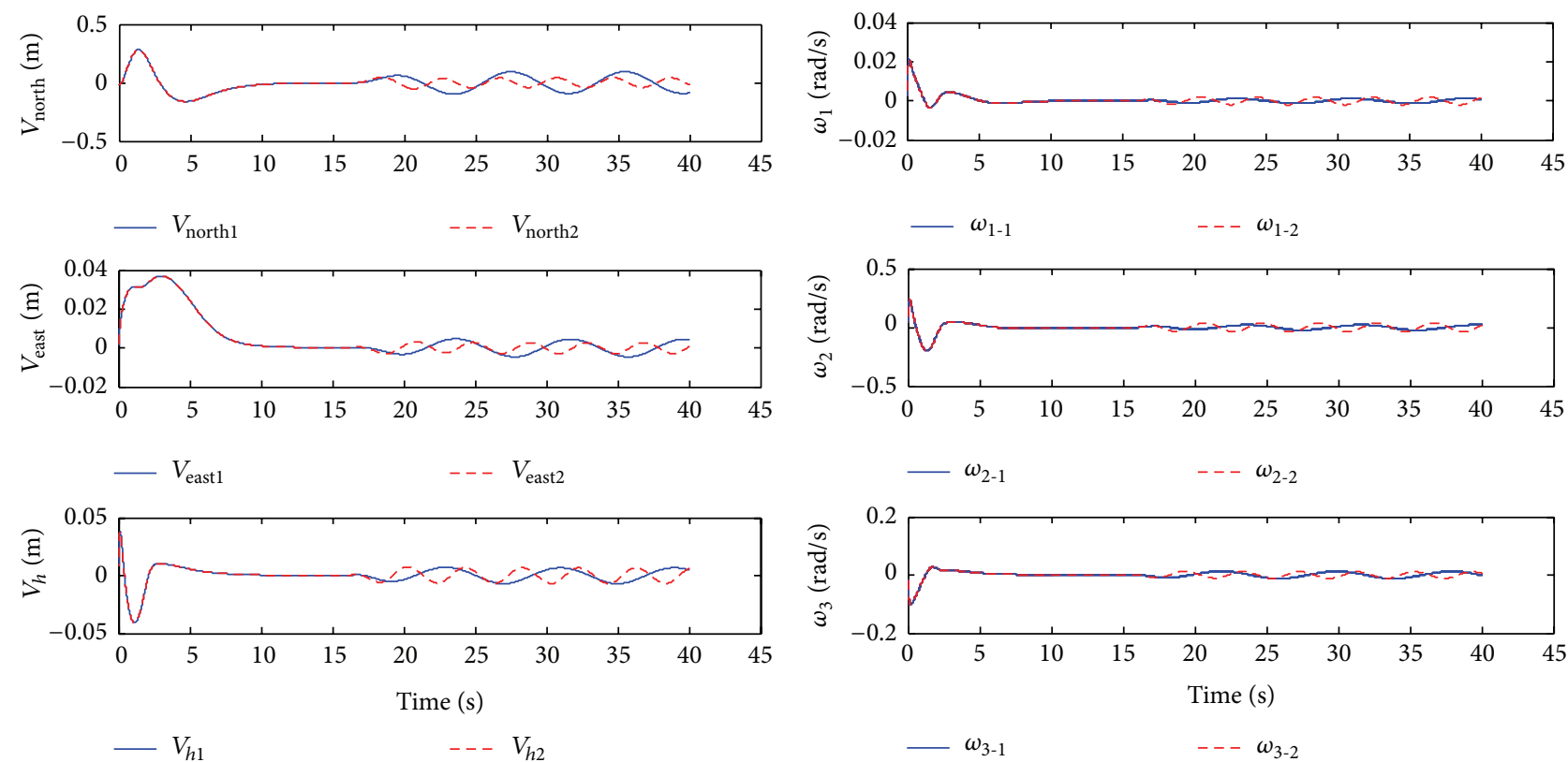

$-V_{h 1}$

$---V_{h 2}$

(d)
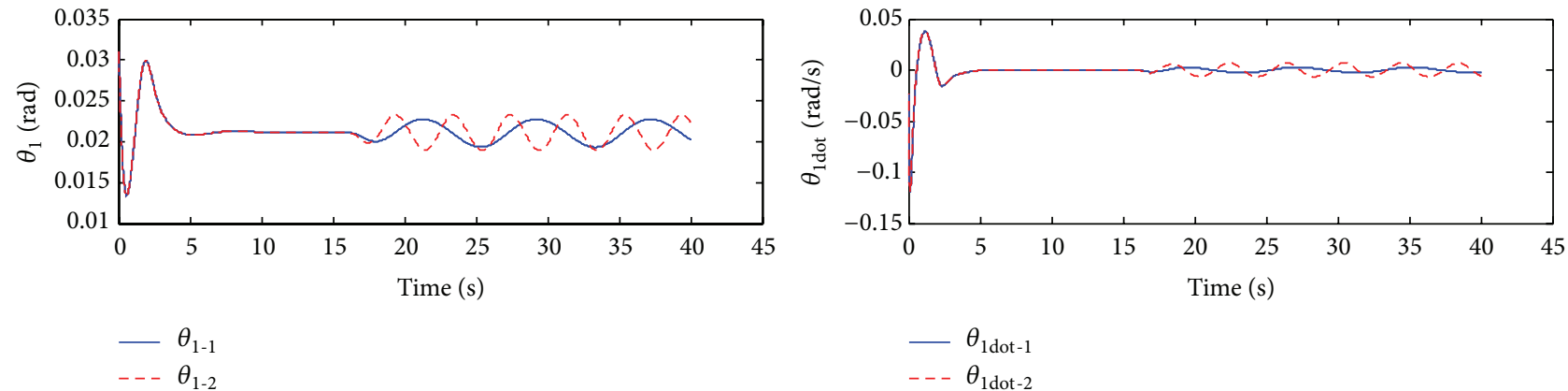

(e)

FIgure 7: Continued. 

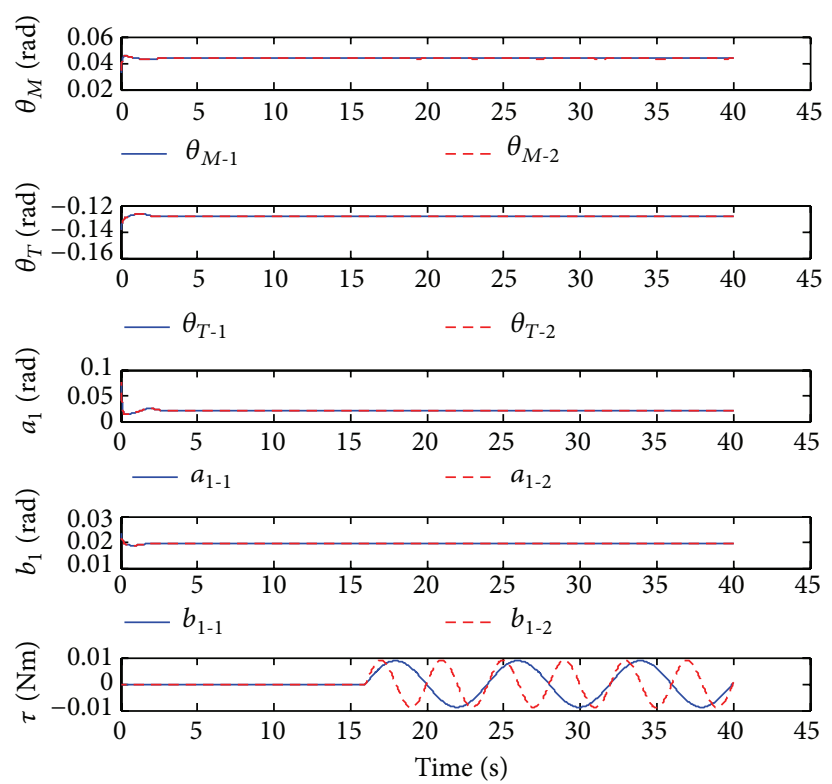

$\tau-1$

$---\tau-2$

(f)

Figure 7: States response with disturbance (49) (blue and solid) and (50) (red and dashed) ${ }^{*}$; the subscript -1 means that the results are with the disturbance (49) and the subscript -2 means that the results are with the disturbance (50).

and $\bar{A}_{17}$ is heavy. Using the following QR parameters in the LQR controller,

$$
\begin{gathered}
Q=\operatorname{diag}(1,1,1,1,1,1,10,10,10,10,10,10,10,10) ; \\
R=\operatorname{diag}(1000,1000,1000,1000,1000),
\end{gathered}
$$

the LQR feedback control law is designed as

$$
\begin{gathered}
u=K \Delta x \\
K=\left[\begin{array}{lllll}
K_{1} & K_{2} & K_{3} & K_{4} & K_{5}
\end{array}\right],
\end{gathered}
$$

where

$$
\begin{aligned}
K_{1} & =\left[\begin{array}{ccc}
0.0097 & -0.0037 & -0.0226 \\
0.0119 & 0.0058 & 0.0210 \\
-0.0232 & -0.0014 & 0.0013 \\
-0.0018 & 0.0308 & -0.0066 \\
0.0149 & -0.0006 & -0.0008
\end{array}\right] \\
K_{2} & =\left[\begin{array}{ccc}
0.0200 & -0.0071 & -0.0291 \\
0.0238 & 0.0116 & 0.0287 \\
-0.0544 & -0.0019 & 0.0031 \\
-0.0042 & 0.0575 & -0.0079 \\
0.0308 & -0.0013 & -0.0016
\end{array}\right] \\
K_{3} & =\left[\begin{array}{ccc}
0.0502 & 0.1489 & 0.1317 \\
-0.0816 & 0.1670 & 0.1279 \\
0.0053 & -0.4953 & -0.3462 \\
-0.3754 & -0.0384 & -0.0293 \\
0.0104 & 0.2401 & 0.0991
\end{array}\right]
\end{aligned}
$$$$
K_{4}=\left[\begin{array}{ccc}
0.0169 & 0.0637 & 0.0751 \\
-0.0226 & 0.0663 & 0.0827 \\
-0.0015 & -0.2717 & -0.0288 \\
-0.1208 & -0.0214 & -0.0058 \\
0.0040 & 0.1085 & 0.0073
\end{array}\right]
$$

$$
K_{5}=\left[\begin{array}{cc}
0.6994 & 0.1310 \\
0.5973 & 0.1125 \\
-4.2835 & -0.8102 \\
-0.3735 & -0.0691 \\
1.1293 & 0.2157
\end{array}\right] \text {. }
$$

With the controller (46), the system can be stabilized with acceptable performance near the trim point, and the simulation results with initial state $(0.1,0,-0.1,0,0,0,0.07$, $0.04,0,0,0,0.03,0)$ are shown in Figure 6.

From Figure 6, it can be seen that a linear LQR controller can stabilize the whole system near the trim point. However, the stabilizing region of LQR is very limited; we have tested that only when the attitude of the whole system satisfies the following conditions (all the initial velocities are set to zeros), the LQR control is effective (46):

$$
\begin{aligned}
& -0.1389 \leq \theta \leq 0.3911 \\
& -0.6731 \leq \phi \leq 0.5469 \\
& -0.0619 \leq \theta_{1} \leq 0.0611 .
\end{aligned}
$$

Now in the next simulation, two periodic sinusoidal signals, as disturbances with different frequencies, are added 


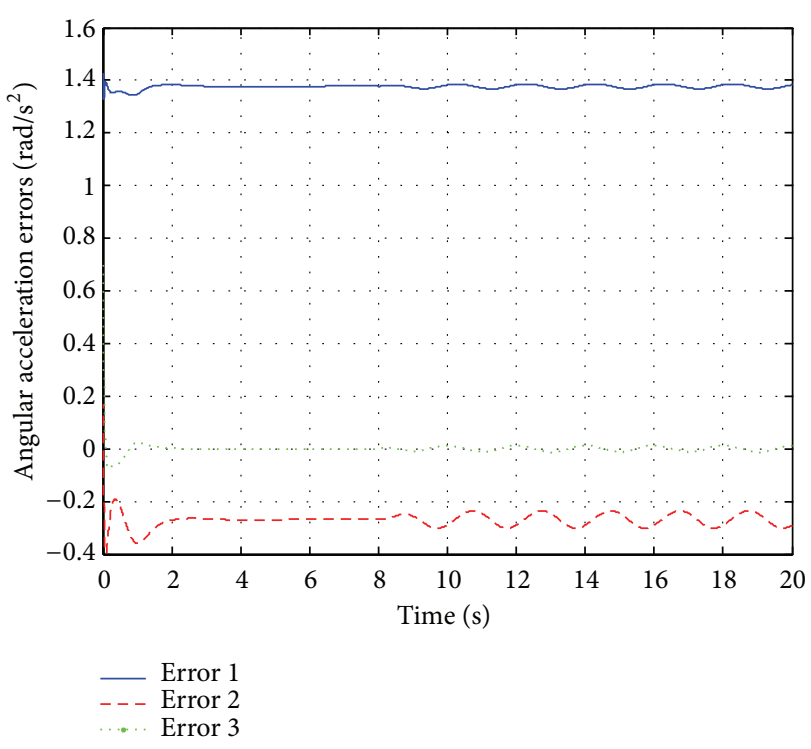

(a)

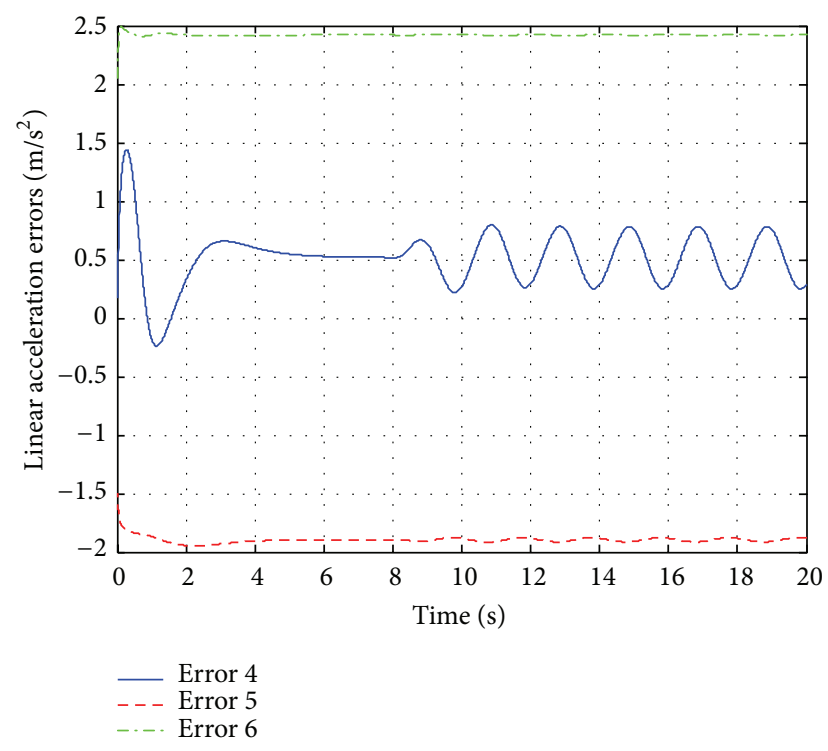

(b)

FIGURE 8: Decoupling between RFR and manipulator.

to the input of the manipulator to test motion influence of the manipulator on the whole system:

$$
\begin{aligned}
& d_{1}=0.01 \sin (0.5 \pi t) \\
& d_{2}=0.01 \sin (1.0 \pi t) .
\end{aligned}
$$

The results are as in Figure 7.

Simultaneously, in order to test the coupling between the RFR and the manipulator, the linear accelerations and the angular accelerations of the new RF-MJM system are compared to the helicopter system with the same parameters as in Table 1; that is,

$$
\begin{aligned}
& {\left[\begin{array}{c}
\text { error } 1 \\
\text { error } 2 \\
\text { error } 3
\end{array}\right]=\left[\begin{array}{c}
a_{\mathrm{RF}-\mathrm{MJM}, x} \\
a_{\mathrm{RF}-\mathrm{MJM}, y} \\
a_{\mathrm{RF}-\mathrm{MJM}, z}
\end{array}\right]-\left[\begin{array}{c}
a_{\mathrm{RFR}, x} \\
a_{\mathrm{RFR}, y} \\
a_{\mathrm{RFR}, z}
\end{array}\right] ;} \\
& {\left[\begin{array}{c}
\text { error } 4 \\
\text { error } 5 \\
\text { error } 6
\end{array}\right]=\left[\begin{array}{c}
\dot{\omega}_{\mathrm{RF}-\mathrm{MJM}, x} \\
\dot{\omega}_{\mathrm{RF}-\mathrm{MJM}, y} \\
\dot{\omega}_{\mathrm{RF}-\mathrm{MJM}, z}
\end{array}\right]-\left[\begin{array}{c}
\dot{\omega}_{\mathrm{RFR}, x} \\
\dot{\omega}_{\mathrm{RFR}, y} \\
\dot{\omega}_{\mathrm{RFR}, z}
\end{array}\right] .}
\end{aligned}
$$

The results are given in Figure 8, which presents the extra force and moment exerted on the RFR due to the manipulator and its motion.

\section{Conclusions}

In this paper, the detailed nonlinear dynamics model of a rotor-flying multijoint (RF-MJM) system is constructed through using Euler-Lagrange method. Compared to the rotor-flying vehicle system, the model nonlinearities and complexity of the new RF-MJM are analyzed in detail. Moreover, linear analysis is conducted with respect to the constructed nonlinear model near its trim point, and the influence of the manipulator mass on the system's local performance is researched. Furthermore, LQR controller is designed based on the linearized system model. Finally, simulation results show that (1) a linear LQR controller is able to stabilize the system near steady state and presents acceptable performance; however, (2) the stabilization region of LQR controller is very limited, and the performance of LQR controller is sensitive to the external disturbance. Thus, in the future work, nonlinear and robust control scheme will be researched to overcome the disadvantages of the linear controller.

\section{Conflict of Interests}

The authors declare that there is no conflict of interests regarding the publication of this paper.

\section{Acknowledgment}

This work was supported by the National Natural Science Foundation of China (no. 61305120 and 61035005).

\section{References}

[1] A. Birk, B. Wiggerich, H. Bülow, M. Pfingsthorn, and S. Schwertfeger, "Safety, security, and rescue missions with an Unmanned Aerial Vehicle (UAV): aerial mosaicking and autonomous flight at the 2009 European Land Robots Trials (ELROB) and the 2010 Response Robot Evaluation Exercises (RREE)," Journal of Intelligent and Robotic Systems: Theory and Applications, vol. 64, no. 1, pp. 57-76, 2011. 
[2] C. Bolkcom, "Homeland security: unmanned aerial vehicles and border surveillance," 2004.

[3] G. Cai, B. M. Chen, and T. H. Lee, "An overview on development of miniature unmanned rotorcraft systems," Frontiers of Electrical and Electronic Engineering in China, vol. 5, no. 1, pp. 1-14, 2010.

[4] A. Puri, A Survey of Unmanned Aerial Vehicles (Uav) For Traffic Surveillance, Department of computer science and engineering, University of South Florida, 2005.

[5] K. P. Valavanis, Advances in Unmanned Aerial Vehicles: State of the Art and the Road to autonomy, vol. 33, Springer, 2007.

[6] Y. Q. Bai and H. Liu, "Robust flight control of quadrotor unmanned air vehicles," Journal of Robot, vol. 34, no. 5, pp. 519524, 2012.

[7] M. Bernard and K. Kondak, "Generic slung load transportation system using small size helicopters," in Proceedings of the IEEE International Conference on Robotics and Automation (ICRA '09), pp. 3258-3264, Kobe, Japan, May 2009.

[8] D. Mellinger, M. Shomin, N. Michael, and V. Kumar, "Cooperative grasping and transport using multiple quadrotors," in Distributed Autonomous Robotic Systems, pp. 545-558, Springer, 2013.

[9] P. E. Pounds, D. R. Bersak, and A. M. Dollar, "Grasping from the air: hovering capture and load stability," in Proceedings of the IEEE International Conference on Robotics and Automation (ICRA '11), pp. 2491-2498, IEEE, 2011.

[10] X. Y. Xu and T. Kanade, Space Robotics: Dynamics and Control, Springer, 1993.

[11] Y. Q. He and J. D. Han, "Acceleration-feedback-enhanced robust control of an unmanned helicopter," Journal of Guidance, Control, and Dynamics, vol. 33, no. 4, pp. 1236-1250, 2010.

[12] A. R. Bramwell, D. Balmford, and G. Done, Bramwell's Helicopter dynamics, Butterworth-Heinemann, 2001.

[13] D. H. Shim, Hierarchical control system synthesis for rotorcraftbased unmanned aerial vehicles [Ph.D. thesis], Berkeley, Calif, USA, 2000.

[14] J. Hespanha, "Undergraduate lecture notes on lqg/lqr controller design," in Apuntes Para Licenciatura. UCBS, pp. 1-37, University of California, Santa Barbara, Calif, USA, 2007. 


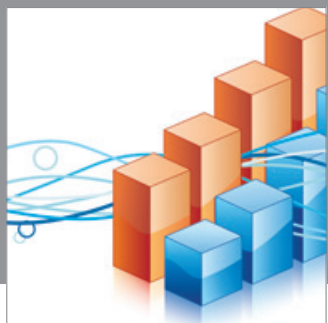

Advances in

Operations Research

mansans

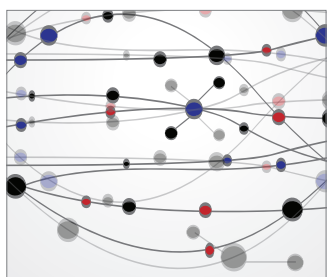

The Scientific World Journal
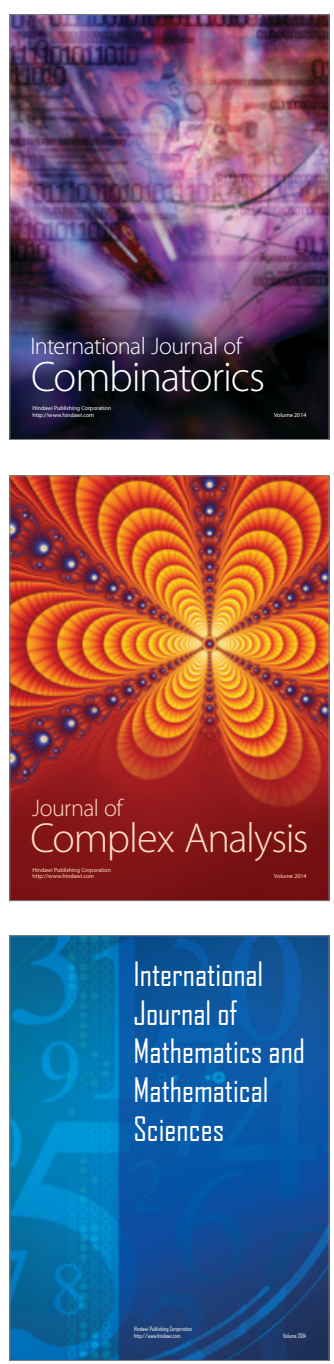
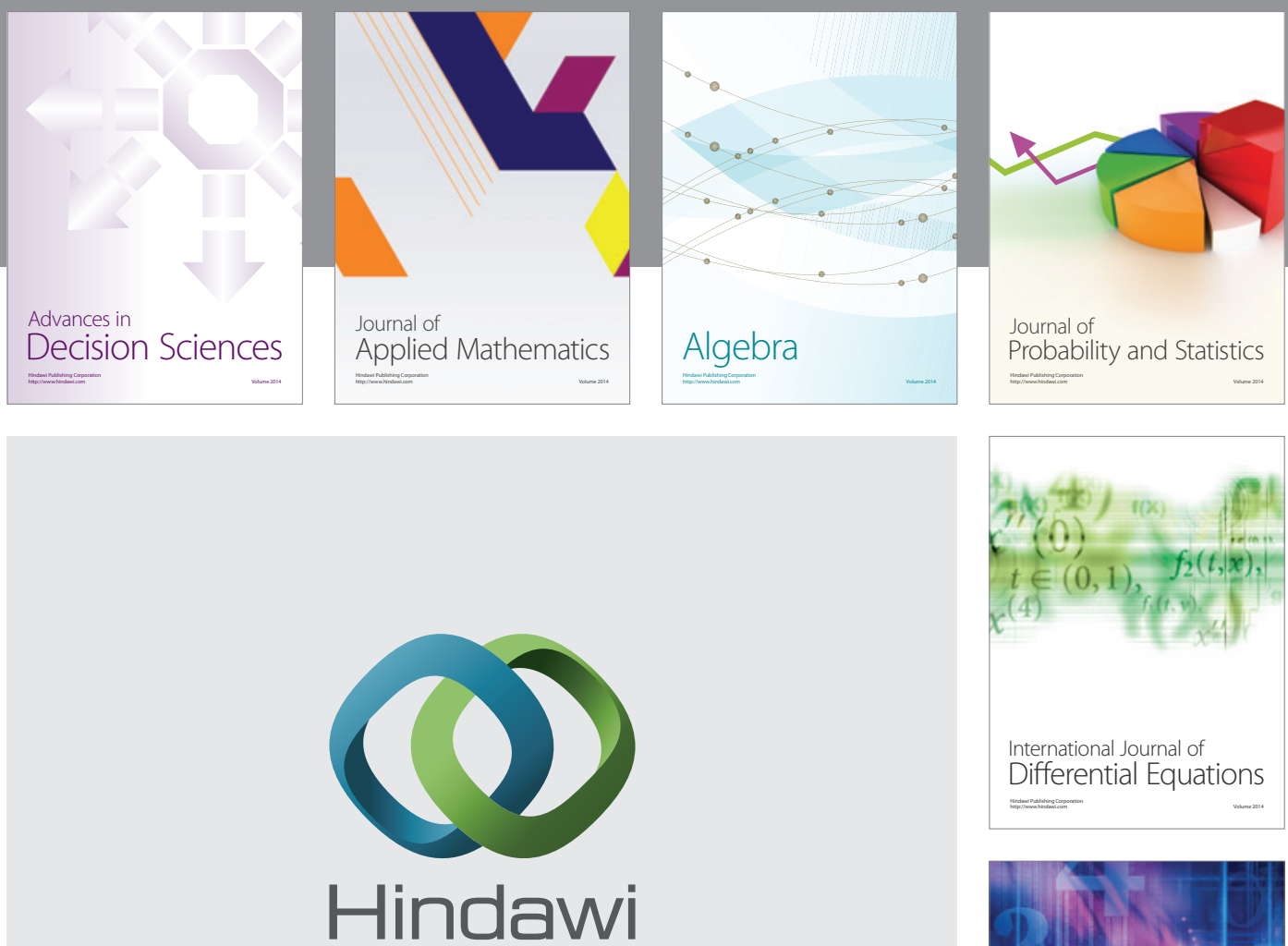

Submit your manuscripts at http://www.hindawi.com
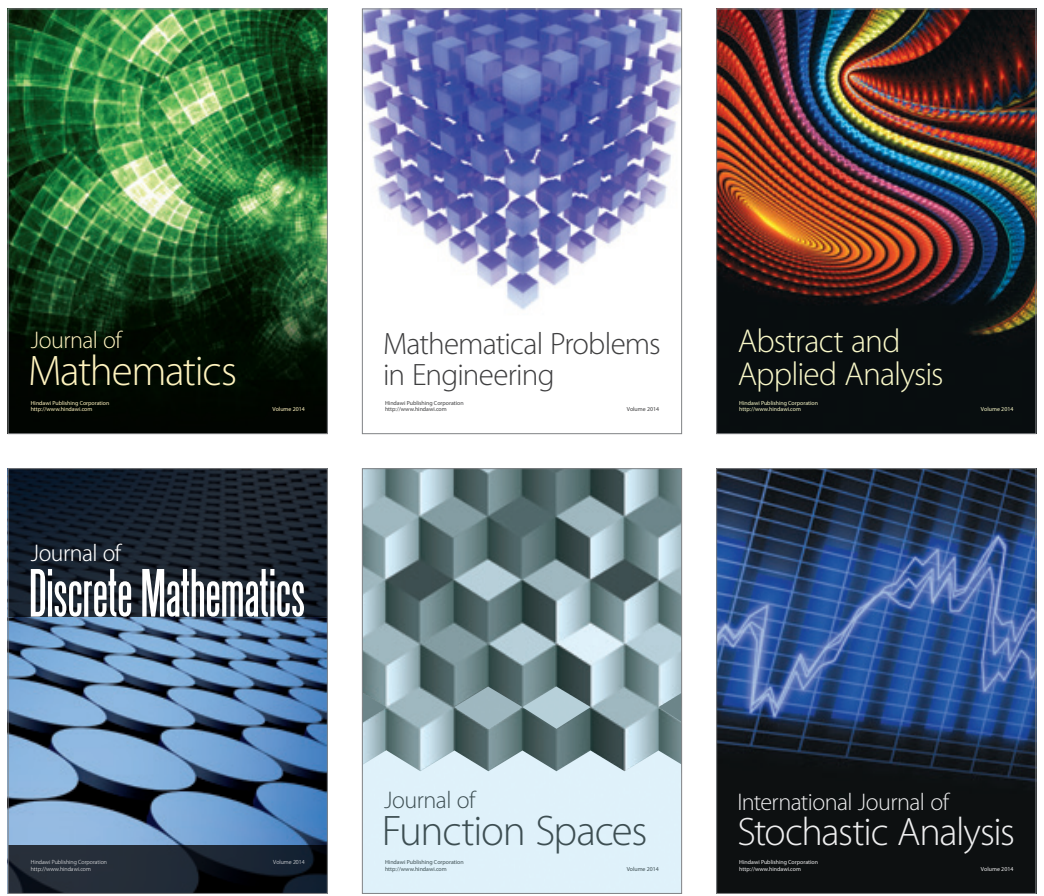

Journal of

Function Spaces

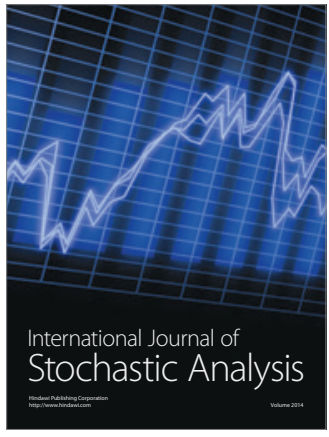

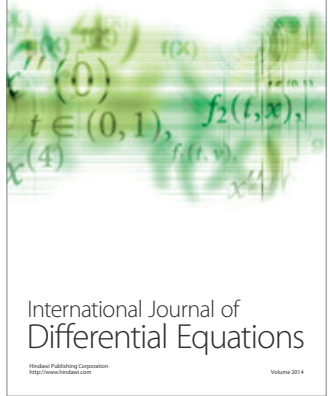
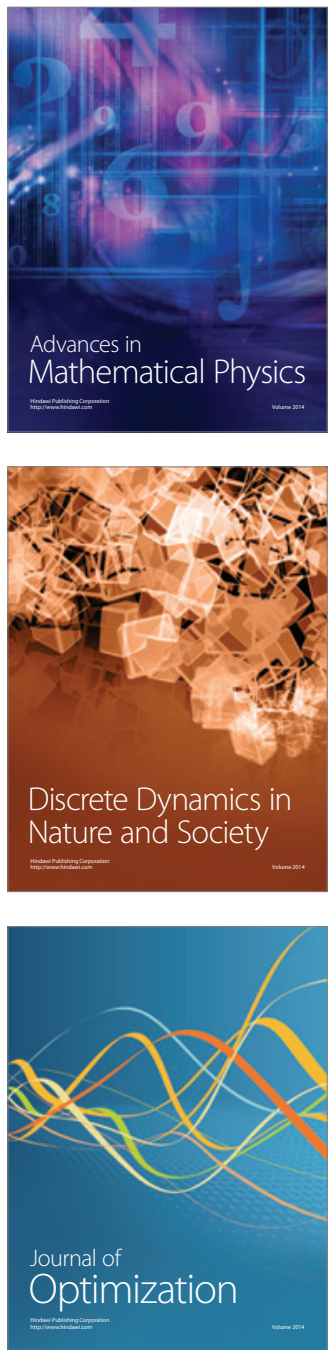